\title{
Cricopharyngeal Myotomy Revisited
}

\author{
${ }^{1}$ Sudhakara M Rao, ${ }^{2}$ Satishchandra T, ${ }^{2}$ PSN Murthy \\ ${ }^{1}$ Associate Professor, Department of ENT and Head and Neck Surgery, Dr PSIMS and RF, Gannavaram, Andhra Pradesh, India \\ ${ }^{2}$ Professor and Head, Department of ENT and Head and Neck Surgery, Dr PSIMS and RF, Gannavaram, Andhra Pradesh, India
}

Correspondence: PSN Murthy, Professor and Head, Department of ENT and Head and Neck Surgery, Dr PSIMS and RF Gannavaram, Andhra Pradesh, India, e-mail: drmurtypsn@gmail.com

\section{ABSTRACT}

Dysphagia due to neuromuscular in coordination is major disability for the patient. Not able to swallow food or liquids inspite of healthy appetite makes the patient most irritable and can lead to psychological problems. Added to the swallowing problem patient also encounters symptoms and signs of laryngeal penetration or aspiration. For these patients, surgical option of cricopharyngeal myotomy offers a very good relief. We describe two cases where CP myotomy could facilitate a good swallow and prevent laryngeal stimulation or penetration and made a significant improvement in the quality of life of the patients.

Keywords: Cricopharyngeal myotomy, Cricopharyngeal spasm, Neurogenic dysphasia, Cricopharyngeus muscle.

\section{INTRODUCTION}

Cricopharyngeal spasm can be a primary or secondary to several neurologic disorders. ${ }^{1} \mathrm{M}$ ainly these are the patients who have a basic neurologic disorder from which they were recovering but present with swallowing and aspiration problems. M ost of these patients are stuck with a permanant NG tube or a PEG for improving the nutritional status. A simple CP myotomy in selected cases can really make a difference in the quality of Iife. $^{9}$

\section{CASE REPORTS}

\section{Case 1}

A 25-year-old male patient presented with history of gradually progressive difficulty in swallowing from the past 6 months. He was investigated for the same complaint 6 months ago by upper GI scopy which revealed normal findings and was given PPIs at that time. One month later he developed an episode of loss of conciousness and found to have tuberculous meningitis with hydrocephalus. He underwent ventriculoperitoneal shunting and kept on A TT. D espite surgery and medication his dysphagia was gradually worsening, more for liquids than solids and with intermittant choking during attempted swall owing and he was unable to take sufficient diet and water inspite of good appetite and thirst. He was irritable in his behavior with relatives and developed talking during sleep. On examination he was looking dull and exhausted, V P shunting scars are present. When he was asked to drink water, he is forcibly pushing bolus dow $n$ into hypopharynx taking time for each swallow and the situation is similar for solids. Indirect laryngoscopy reveal ed pooling of saliva in both pyriform fossa. B arium swallow studies revealed constricted cricopharyngeal area with pooled barium in hypopharynx and little barium going down into esophagus and stomach (Figs 1 A and B). A clinical diagnosis of cricopharyngeal spasm was made with possible differential diagnosis as postcricoid carcinoma.

Hypopharyngoscopy was planned to ruleoutany postcricoid obstructive lesion followed by external cricopharyngeal myotomy in thesame sitting under GA. Hewas given nasogastric feeds for 3 days before surgery. During surgery, hypopharyngoscopy revealed no abnormality of postcricoid area. A nother endotracheal tubewas placed in theesophagus to identify cricopharyngeal sphincter. H orizontal skin crease incision was taken over cricoid level. Subplatysmal flaps wereelevated. D eep fascia was divided along the anterior border of leftsternocleidomastoid and strap muscles retracted medially. O mohyoid was identified and di vided. B y inflating the cuff of endotracheal tube present in esophagus, cricopharyngeus muscle was made prominent and it was cut with number 15 blade throughout its length from thyropharyngeusto circular musclecoat of esophagus till the mucosa was seen bulging out. Endotracheal tube from esophagus was removed and wound closed with drains. He was given nasogastric feeds for 3 days postoperatively, and when he was asked to drink after 3 days he was very comfortable and he has completed full glass of water in onego. Postoperative contrast pharyngogram showed a freefl ow of barium through pharyngoesophageal segment (Fig. 2).

\section{Case 2}

A 45-year-old lady presented with absolute dysphagia from the past one month. She was operated for left cerebellopontine angle tumor 1 month back and she was on nasogastric feeds for the last 3 weeks. She developed left vocal cord and palatal paralysis as a complication of surgery. She has not recovered from dysphagia after 3 w eeks. She wanted al ternative for nasogastric tube. Her X-ray barium swallow revealed constricted cricopharyngeal area with pooled barium in hypopharynx and no barium passed beyond the sphincter.

She was offered external cricopharyngeal myotomy and was improved dramatically from her dysphagia, 1 week following surgery.

\section{DISCUSSION}

The cricopharyngeus muscle is a true sphincter composed of striated muscle arising from the lateral borders of the cricoid 
cartilage, the muscle fibers form a sling around the wall of the superior aspect of the cervical esophagus. The cricopharyngeus muscle is bordered superiorly by the thyropharyngeus muscle and merges inferiorly with the muscular layers of the cervical esophagus. The muscle is innervated primarily by the vagus nerve, both by branches from the pharyngeal plexus and by neuronal branches from the recurrent laryngeal nerve. ${ }^{1}$ Therefore, the recurrent laryngeal nerves lie in close proximity within the surgical field.

Cricopharyngeal achalasia may be primary or secondary. Primary cricopharyngeal achal asia implies that the abnormal ity that leads to the persistent spasm or failure of relaxation of the cricopharyngeus muscle is confined to the muscle, with no underlying neurologic or systemic cause. ${ }^{2}$ This primary group can be further subdivided into primary cricopharyngeal achalasia with no underlying cause (i.e. idiopathic) or cricopharyngeal achalasia caused by intrinsic disorders of the cricopharyngeus muscle (e.g. polymyositis, muscular dystrophy, hypothyroidism, inclusion body myositis). In many instances, the cricopharyngeal spasm may be secondary to neurologic disorders, such as polio, oculopharyngeal dysphagia, stroke and amyotrophic lateral sclerosis (A LS). Peripheral neurologic disorders, such as diabetic neuropathy, myasthenia gravis, and peripheral neuropathies, can also cause cricopharyngeal dysfunction. ${ }^{3,4}$ A nother important cause for cricopharyngeal spasm is its assosiation with partial laryngectomy status.

The clinical presentation of patients with cricopharyngeal achalasia may be quite variable. Most patients primarily experience food sticking or catching in the lower third of the neck. These patients often point to the cricoid region in their description of the dysphagia. Patients may also experience accompanying symptoms of heartburn, choking and pain with swallowing. Less common symptoms include dysphonia, a globus sensation, and pressure in the neck during deglutition with intermittant choking from laryngeal spillover. Symptoms have often been present for months to years. In cases related to neurologic causes, the diagnosis of cricopharyngeal achalasia may postdate the neurologic event or diagnosis by several months or years. Both our patients presented with a great difficulty in swallowing both for solids and liquids, associated with neurologic event.

Cricopharyngeal myotomy may be considered if conservative measures fail and radiologic evidence exists on the video fluoroscopic swallowing study (VFSS) of cricopharyngeal dysfunction demonstrating hesitation of bolus passage. During VFSS, the patient is asked to swallow barium-coated material of different varieties, including liquid barium, while cineradiographic data are collected as the bolus is followed from swallow initiation in the oral cavity to the delivery of the bolus to the stomach. The classic finding on a diagnostic VFSS of cricopharyngeal achalasia is the presence of the horizontal bar (often called the cricopharyngeal bar) at the level of the cricoid cartilage. This makes a posterior indentation in the barium column that persists throughout the swallow. The VFSS also provides information about the presence or absence of laryngeal spillover, aspiration, nasopharyngeal regurgitation and pharyngeal stasis, each of which may accompany cricopharyngeal dysfunction. ${ }^{7,15}$ We largely depend on clinical presentation and findings on $\mathrm{X}$-ray barium swallow for the diagnosis as they were classical to diagnose the condition.

Cricopharyngeal dysfunction has been largely refractory to medical management, including therapy with muscle relaxants. B otulinum toxin injection into the cricopharyngeus muscle has recently been explored as a possible therapeutic intervention. Although experience with botulinum toxin in this clinical entity is rather limited, it may serve two useful purposes. First, in patients in whom the diagnosis of cricopharyngeal achalasia may be in question, botulinum toxin treatment can be used as a trial of therapy. If the patient's dysphagia symptoms resolve after botul inum toxin injection, the diagnosis of cricopharyngeal achalasia is confirmed, and subsequent cricopharyngeal myotomy may be deemed appropriate. In addition, patients who are medically infirm and cannot undergo external cricopharyngeal myotomy may be considered for botulinum toxin therapy. Unfortunately, the administration of the botulinum toxin into the cricopharyngeus is technically difficult and somew hat uncomfortable for the patient. Furthermore, because the effective botul inum toxin is temporary, patients are required to undergo repeat injections to maintain therapeutic efficacy. Finally, inadvertent injection outside the cricopharyngeus may result in temporary paralysis of the laryngeal musculature, causing dysphonia and, rarely, aspiration. 8,13,14

There are mainly two surgical approaches for treatment of cricopharyngeal dysfunction. The classic approach is the external cricopharyngeal myotomy technique., ${ }^{9,10,12}$

Recently, investigators have been exploring a transoral endoscopic laser approach for cricopharyngeal myotomy. However, only limited case series have been published, and follow-up data are rather limited. Initial data suggest that this technique may have applications when the technique is refined and longer follow-up is available. A Iso, an endoscopic approach has the advantages of avoiding an external neck incision and, to some degree, less risk to surrounding structures. ${ }^{5,6,11}$

Because patients have often had dysphagia for some time prior to surgical intervention, optimizing their nutritional and heal th status prior to surgical intervention is important. In certain cases, particularly with significant weight loss prior to surgery, a temporary nasogastric feeding tube or PEG may be required to administer nutritional supplementation to increased serum protein levels. In those patients with a history of preoperative aspiration, pulmonary insufficiency must al so be corrected with appropriate medical consultation. We have given nasogastric feeding for first patient as he was deprived of food for long time.

The most common complication of external cricopharyngeal myotomy is inadvertent entry into the lumen of the cervical esophagus. Although thoroughly sectioning the entire cricopharyngeus muscle is important, the margin for error is 
small because the esophageal submucosa and mucosa are very thin. If the cervical esophageal lumen is entered, repair it with a watertight closure in a single layer. A void a double-layer closure because it may predispose the patient to stenosis in this area, defeating the original purpose of the procedure. In the setting of an inadvertent esophagotomy, maintain wound drainage and monitor the patient for evidence of salivary fistula through the drain site. $M$ aintain perioperative and postoperative antibiotics to cover oral flora. If no evidence of air leakage or salivary fistula is present, the patient may resume a gradually advancing diet on postoperative day 3 , with anticipated discharge 1 to 2 days later. ${ }^{10}$

Other complications may occur during cricopharyngeal myotomy. A relatively uncommon occurrence is injury to the recurrent laryngeal nerve, which most often manifests as hoarseness after extubation and most often is due to a stretch injury to the nerve. M ost injuries such as this resolve with time; however, recovery may require several months. During the recovery phase, monitor the patient for aspiration and pulmonary complications. ${ }^{10,12} \mathrm{~W}$ e did not encounter any of the complications for either case.

\section{CONCLUSION}

Cricopharyngeal spasm can occur both primarily or secondary to many neurological conditions including the commonly occuring stroke. Patients present with variable degrees of difficulty in swallowing, i.e. from a feeling of sticking of food in throat to absolute dysphagia. This condition can be diagnosed based on history, clinical examination and results of radiologic investigations. This condition is usually refractory to medical treatment. Surgery offers great improvement of their symptoms, the standard procedure being external cricopharyngeal myotomy. W e have to improvethe nutritonal status of the patient if necessary before taking for surgery by nasogastric feeding.
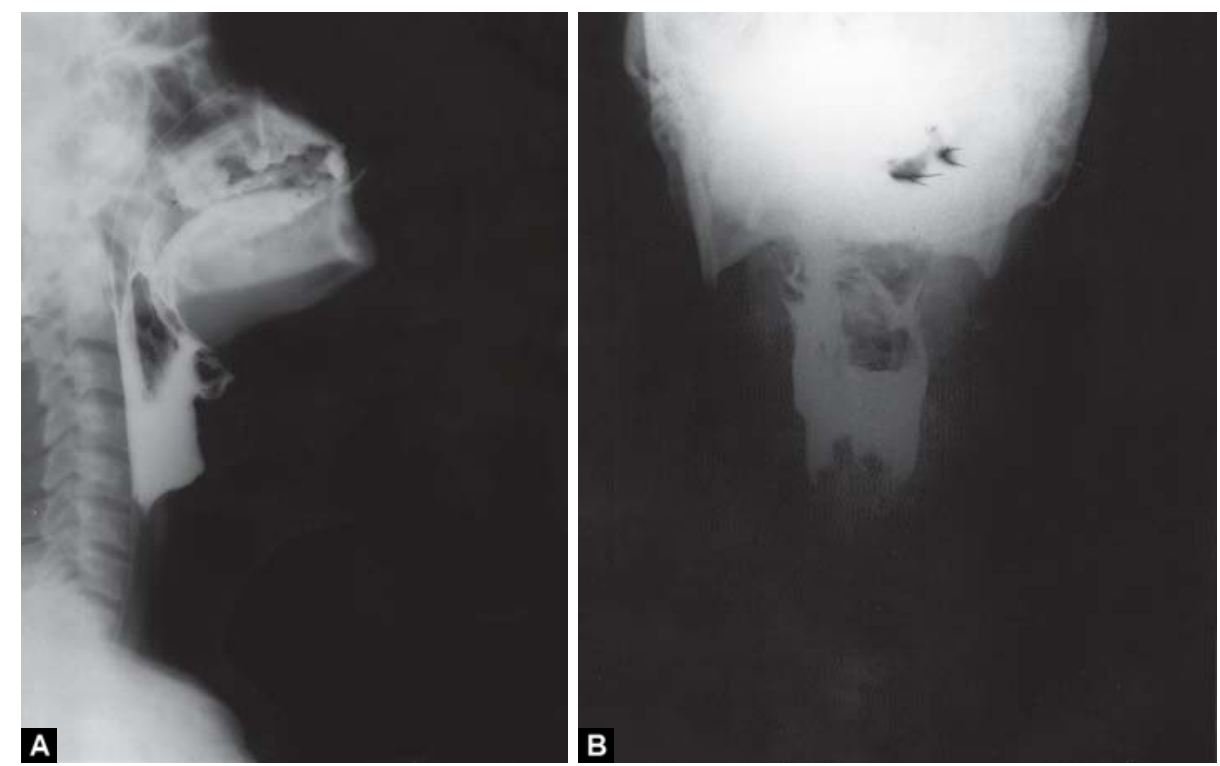

Figs 1A and B: Preoperative barium swallow

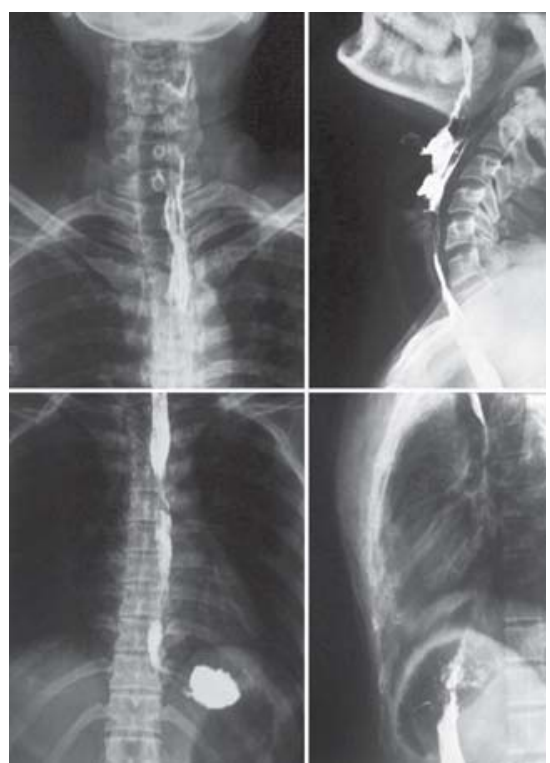

Fig. 2: Postoperative photograph 


\section{REFERENCES}

1. Wilson JA, Pryde A, A llan PL, M aran A G. Cricopharyngeal dysfunction. Otolaryngol Head Neck Surg Feb 1992; 106(2):163-68.

2. Fujimoto $Y$, Hasegawa $Y, Y$ amada $H$, A ndo A, Nakashima $T$. Swallowing function following extensive resection of oral or oropharyngeal cancer with laryngeal suspension and cricopharyngeal myotomy. Laryngoscope Aug 2007;117(8): 1343-48.

3. Coiffier L, Perie S, Laforet P, Eymard B, St Guily JL. Longterm results of cricopharyngeal myotomy in oculopharyngeal muscular dystrophy. Otolaryngol Head Neck Surg Aug 2006;135(2):218-22.

4. Oh TH, Brumfield KA, Hoskin TL, K asperbauer JL, Basford JR. Dysphagia in inclusion body myositis: Clinical features, management and clinical outcome. Am J Phys Med Rehabil Nov 2008;87(11):883-89.

5. Pitman $\mathrm{M}, \mathrm{W}$ eissbrod $\mathrm{P}$. Endoscopic $\mathrm{CO}_{2}$ laser cricopharyngeal myotomy. Laryngoscope J an 2009;119(1):45-53.

6. Dauer E, Salassa J, I uga L, K asperbauer J. Endoscopic laser vs open approach for cricopharyngeal myotomy. Otolaryngol Head Neck Surg M ay 2006;134(5):830-35.

7. Baredes S, Shah CS, K aufman R. The frequency of cricopharyngeal dysfunction on videofluoroscopic swall owing studies in patients with dysphagia. A m J Otolaryngol May-Jun 1997;18(3):185-89.
8. Blitzer A, B rin M F. U se of botulinum toxin for diagnosis and management of cricopharyngeal achalasia. Otolaryngol Head Neck Surg M ar 1997;116(3):328-30.

9. Bonavina $L, K$ han NA, DeM eester TR. Pharyngoesophageal dysfunctions. The role of cricopharyngeal myotomy. A rch Surg M ay 1985;120(5):541-49.

10. K elly JH. M anagement of upper esophageal sphincter disorders: Indications and complications of myotomy. A m J M ed M ar 6 2000;108 Suppl 4a:43S-46S.

11. L awson G, Remacle M , J amart J, Keghian J. Endoscopic $\mathrm{CO}_{2}$ laser-assisted surgery for cricopharyngeal dysfunction. Eur A rch Otorhinolaryngol Oct 2003;260(9):475-80.

12. Lindgren $\mathrm{S}$, Ekberg 0 . Cricopharyngeal myotomy in the treatment of dysphagia. Clin Otolaryngol Allied Sci Jun 1990;15(3):221-27.

13. M oerman M B. C ricopharyngeal botox injection: Indications and technique. Curr Opin Otolaryngol Head Neck Surg Dec 2006;14(6):431-36.

14. Parameswaran M S, Soliman A M . Endoscopic botulinum toxin injection for cricopharyngeal dysphagia. Ann Otol Rhinol Laryngol Oct 2002;111(10):871-74.

15. M uñoz A A , Shapiro J, Cuddy LD, M isono S, B hattacharyya N . $\checkmark$ ideofluoroscopic findings in dysphagic patients with cricopharyngeal dysfunction: B efore and after open cricopharyngeal myotomy. A nn Otol Rhinol Laryngol J an 2007; 116(1):49-56. 